\title{
Performance of Fattening Pigs in a Farm Infected with Both Porcine Reproductive and Respiratory Syndrome (PRRS) Virus and Porcine Circovirus Type 2 Following Sow and Piglet Vaccination with an Attenuated PRRS Vaccine
}

\author{
S. K. Kritas ${ }^{1,3}$, C. Alexopoulos ${ }^{2}$, C. S. Kyriakis ${ }^{2}$, E. Tzika² and S. C. Kyriakis ${ }^{2}$ \\ Addresses of authors: ${ }^{1}$ Department of Microbiology and Infectious Diseases, ${ }^{2}$ Clinic of Productive Animal Medicine, School of \\ Veterinary Medicine, Aristotle University of Thessaloniki, 54124 Thessaloniki, Macedonia, Greece; ${ }^{3}$ Corresponding author: \\ Tel.: + 302310999940 or + 30 6937151122; fax: + 30 2310999959; E-mail: skritas@vet.auth.gr \\ With 3 tables \\ Received for publication August 22, 2006
}

\begin{abstract}
Summary
The purpose of the study was to investigate whether, on farms with both post-weaning multisystemic wasting syndrome (PMWS) and porcine reproductive and respiratory syndrome (PRRS), the PRRS vaccination of sows and their fattening pigs protects against these syndromes. In a farrow-to-finish pig farm with a history of PRRS and PMWS, 200 gilts and sows were allocated to one of two groups of equal size. The first group (C-sow group) was used as untreated controls, while the animals of the second group (V-sow group) were vaccinated with live Porcilis PRRS $^{\circledR}$ vaccine. At the next weaning, all piglets of half the sows of the $\mathrm{C}$ sow group were vaccinated once at 35 days of age with the vaccine (CV group), while the offspring of the other half of the unvaccinated sows were left unvaccinated (CC group). Similarly, the offspring of half the sows of the $\mathrm{V}$ sow group were vaccinated (VV group), while those of the other half of the vaccinated sows were left unvaccinated (VC group). No significant differences in morbidity were observed between the groups during the nursery and finishing phases, while morbidity in the growers was significantly reduced in the $\mathrm{CV}$ - and VV-groups $(P<0.05)$. Growers' mortality was significantly reduced after piglet vaccination when compared with unvaccinated pigs of unvaccinated dams $(P<0.05)$. Average daily gain and feed conversion ratio were significantly improved in vaccinated piglets compared with those in the unvaccinated groups $(P<0.05)$.
\end{abstract}

\section{Introduction}

Porcine reproductive and respiratory syndrome (PRRS) is a viral disease caused by PRRS virus (PRRSV), a member of the family Arteriviridae. It is characterized by reproductive disorders in breeding pigs, and respiratory malfunction mainly apparent in growing and fattening pigs (Keffaber, 1989; Polson et al., 1992; Meredith, 1995). The severity of PRRSV infection can vary widely, ranging from an almost complete lack of clinical signs to devastating outbreaks of reproductive and respiratory disease (Benfield et al., 1999). Such variation also depends on the origin of the infective strain as phylogenetic and pathogenetic differences have been described between European and American clusters, but also within each cluster (Wensvoort et al., 1992; Murakami et al., 1994; Magar et al., 1995; Dea et al., 1996; Labarque et al., 2004). Fundamental to the control of PRRS is achievement of stability of the virus circulation in the breeding herd as for instance by not introducing naïve gilts or by keeping a constant level of immunity (Dee and Philips, 1999; Dee, 2003). One way of achieving this is by vaccination of the breeding herd. It appears that vaccination of the fattening pigs may also contribute to the stabilization process. Protection conferred by the vaccines can be variable, basically due to strain differences, but homogenous vaccine strains nonetheless appear to provide good protection (Labarque et al., 2004).

Post-weaning multisystemic wasting syndrome (PMWS) is another recently studied disease entity of swine associated with porcine circovirus type 2 (PCV2). It is mostly observed in 5- to 12-week-old weaned pigs causing gradual loss of bodyweight, pallor, dyspnoea and lymph node swelling with high levels of morbidity, and high case fatality in acute cases (Lukert and Allan, 1999).

In the field, PRRSV and PCV2 are quite often found in the same population (Rose et al., 2003; Ellis et al., 2004; Wellenberg et al., 2004). Experimental co-infection of conventional pigs with the two agents has shown that PRRSV is the most damaging of the two, causing severe disease, and that PMWS can be reproduced in its most severe form when there is coinfection with PRRSV (Rovira et al., 2002). However, the effect of vaccination against PRRSV in combined field infections is not yet known.

The aim of this study was to assess the efficacy of Porcilis PRRS ${ }^{\circledR}$, a live vaccine prepared from a European strain of PRRSV, on the health status and productivity of fattening pigs on a farm infected by both PRRSV and PCV2. 


\section{Materials and Methods}

\section{Trial farm}

The trial was performed on an all-in, all-out farrow-to-finish farm of 250 sows, producing approximately 4300-4500 fattening pigs per year. The farm was situated in an area of high pig density, affected by both PRRSV and PCV2 (Filippiada, Preveza, Greece). For at least 1 year prior to the start of the trial, the farm was diagnosed as PRRS positive, based on clinical signs (increased returns to oestrus, small litters, weak piglets and increased piglet mortality) and serology [specific antibody detecting immunoperoxidase monolayer assay (IPMA) technique as described by Wensvoort et al. (1991)].

The diagnosis of PMWS was confirmed 1 month prior to the start of the trial by specific clinical signs (wasting, apparent lymphadenopathy, jaundice, pallor and tachypnea) in pigs at 7-11 weeks of age and by immuno-labelling techniques in tissues from affected pigs (immuno-histochemistry for detecting the PCV2 antigen and in situ hybridization for nucleic acid), as well as lymphoid depletion in lymphnodes (Dr S. Kennedy, Veterinary Sciences Division, Department of Agriculture and Rural Development, Belfast, UK).

Weaning took place weekly, at the age of $26 \pm 2$ days, in batches of 85 (75-95) weaners in the flat deck unit, and the piglets were allocated to pens of 10-12 pigs according to sex and weight. At 63 days of age, the pigs were moved into the growing/finishing house, in pens of 10-12 animals, where they remained until slaughter at the age of 23-24 weeks (95-100 kg body weight). Growing lasted 7 weeks, and finishing 7-8 weeks depending on the feed offered.

The temperature and ventilation of all the houses was controlled automatically. Farm personnel cleaned each pen daily, and when empty, each pen was thoroughly cleaned and disinfected, and then not re-stocked for 2-3 days.

The pigs were provided a home-mixed feed based on corn/ barley/wheat-soya meal, depending on the season. No antimicrobials and/or growth promoters were used in any diet during the trial.

All breeding pigs were vaccinated against Aujeszky's disease virus (ADV), swine influenza virus-SIV, parvovirus (PPV), atrophic rhinitis (AR), Escherichia coli, Clostridium perfringens and Erysipelothrix rhusiopathiae (SE). All fattening pigs were vaccinated against Mycoplasma hyopneumoniae at 7 and 21 days of age, and against ADV and SIV at 90 days of age. All sows were treated with a single ivermectin injection 14 days prior to farrowing, for the control of endo/ectoparasites. Ivermectin was also administered to the boars twice a year. Routine Leptospira spp. serology was undertaken on blood samples from pigs of all ages. Routine mycotoxicological analysis (zearalenone, aflatoxins $B_{1}, B_{2}, G_{1}, G_{2}$, deoxynivalenol (DON), 3-acetyl-DON, 15-acetyl-DON, nivalenol) was carried out on all raw feed materials by the Veterin S.A. laboratory (Aspropyrgos, Attiki, Greece).

\section{Vaccines used in the trial}

The attenuated DV strain of PRRSV (Porcilis PRRS; Intervet, Boxmeer, the Netherlands) originating in Europe was used. A single intramuscular dose contains $\geq 10^{4.0}$ TCID $_{50}$ suspended in $2 \mathrm{ml}$ of a tocopherol-based adjuvant (Diluvac Forte; Intervet).

\section{Experimental protocol}

In a previous study performed in the same trial farm (Alexopoulos et al., 2005), two treatment groups, one with 100 Porcilis PRRS-vaccinated sows (V group) and another with 100 non-vaccinated sows (C group) were observed for one reproductive cycle. Sows were equally allocated in these groups according to their bodyweight and their parity. They were weekly entering the farrowing rooms alternately by group e.g. they were housed separately (Alexopoulos et al., 2005). The weaner pigs of these sows/gilts were further used in the current study, in 20 sequential weekly batches.

The piglets of 50 litters of the $\mathrm{C}$ group of sows and of 50 litters of the $\mathrm{V}$ group of sows were left untreated (CC- and $\mathrm{VC}$-groups of piglets respectively), while the pigs of the rest of the 50 litters of each of the $\mathrm{C}$ - and $\mathrm{V}$-groups of sows, received a single intramuscular dose of Porcilis PRRS vaccine at roughly 5 weeks old. The piglets were ear-marked and were grouped so that, all of the animals of one pen originated from the same group of sows, and remained in the same group up to the end of the experiment.

At the end of the trial, the $\mathrm{C}$ group comprised 662 pigs, and the $\mathrm{V}$ group 826 piglets, 1488 in total.

\section{Parameters recorded and calculated}

Blood samples from 20 sows and 20 finishers had been collected 1 month before the start of the trial and examined for antibodies against a European PRRSV by indirect immunofluorescent assay in US- or EU-type PRRSV infected MA104 cells essentially as described by Yoon et al. (1995). A similar sampling and examination had been repeated 4 months after the start of the trial with 10 samples from each group of sows and from 20 finishers at the slaughter age.

During the trial, all pigs were almost daily monitored for signs of disease (loss of weight, visible lymphadenopathy of inguinal lymphnodes, colour of skin and mucosae, respiratory and enteric disorders, death) by the standard personnel of the farm including a veterinarian.

Ten pens per group were selected so that each batch contained an equal distribution by sex. Thus a total of 329 specifically identified pigs (65 from CC group, 82 from $\mathrm{CV}$ group, 82 from VC group and 100 from VV group) were included. All piglets of selected pens were weighed individually at 26 days (weaning); 63 days (end of nursery period); 112 days (end of grower period) and 161 days (end of finisher period). Feed consumption was recorded weekly, per pen, and the average daily gain (ADG), the average daily feed intake (ADFI) and the feed conversion ratio (FCR) were calculated.

Faecal material obtained from all diarrhoeic pigs was submitted for laboratory examination to determine the presence of pathogens (coccidia, enterobacteriacae) essentially as described elsewhere (Kritas et al., 2003).

\section{Statistical analysis}

Statistical analysis was carried out using the SAS Statistical Package ('The SAS ${ }^{\circledR}$ System' release 6.12 for Windows 1996; SAS Institute Inc., Cary, NC, USA). Each recorded parameter per group was subjected to analysis of variance using the General Linear Models (GLM) procedure, with pen and sex as co-variants. The Duncan's multiple range test was 
used as the comparison test, to determine the statistical difference between groups. The level of significance was set at $P=0.05$. Pearson chi-square analysis was used to distinguish differences between groups for those parameters expressed as frequencies.

\section{Results}

The serological examination of the blood samples from sows and finishing pigs collected 1 month prior to the start of the trial had shown that $>80 \%$ of those tested were seropositive for antibodies against a European PRRSV strain. The serology from sows and finishers 4 months after the start of the trial had shown prevalence of a European PRRSV strain in $>80 \%$ of the animals in both subpopulations of sows and in the finishers.

As observed in both nursery and growers' stage, morbidity was significantly lower in the PRRS-vaccinated pigs (CV- and VV-groups) compared with the CC group, while that of the VC group was at an intermediate level (Table 1). In all groups, morbidity in the growing period was twice that observed in the nursery period (Table 1).

The first clinical cases were observed at the end of the nursery stage and peaked during the growing stage. Wasting was observed in $>65 \%$ of sick animals in the nursery phase and $95 \%$ of sick animals in the growing phase. Clinically visible inguinal lymphadenopathy was observed at frequencies ranging between $45 \%$ and $57 \%$ of the sick animals during the nursery stage, and between $67 \%$ and $77 \%$ of the sick animals during the growing stage. Pallor was observed in $20-57 \%$ of the sick animals during the nursery and growing stages, while the frequency of jaundice and unclassified diarrhoea occurred less frequently (usually $<20 \%$ of the sick animals). During the finishing stage, cough and sometimes, soft feces were indicative of enzootic pneumonia and ileitis, respectively, in affected pigs.

PRRS-vaccinated pigs (CV- and VV-groups) showed a significantly reduced frequency of wasting compared with the CC group, in both nursery and growing stages, while the pattern of lymphadenopathy during the growing stage was similar $(P<0.05)$. With respect to these signs, the frequency in the pigs of the $\mathrm{VC}$ group did not differ significantly from either the CC group or the CV-and VV-groups. Pallor was markedly less frequent in growers of the VV group compared with CC- and CV-groups.

Table 1. Morbidity (loss of weight, visible lymphadenopathy of inguinal lymphnodes, colour of skin and mucosae, respiratory and enteric disorders, other) across the experimental groups

\begin{tabular}{|c|c|c|c|c|}
\hline & \multicolumn{4}{|c|}{ Number of sick pigs/number of total pigs $(\%)$} \\
\hline & $\mathrm{CC}^{*}$ & $\mathrm{CV}$ & $\mathrm{VC}$ & VV \\
\hline NS** & $35 / 282(12.4)^{\mathrm{a}}$ & $21 / 380(5.5)^{\mathrm{b}}$ & $31 / 399(7.8)^{\mathrm{ab}}$ & $14 / 427(3.3)^{\mathrm{b}}$ \\
\hline GS & $70 / 277(25.3)^{\mathrm{a}}$ & $42 / 376(11.2)^{b}$ & $63 / 394(16.0)^{\mathrm{ab}}$ & $34 / 426(8.0)^{\mathrm{b}}$ \\
\hline FS & $9 / 245(3.7)^{\mathrm{a}}$ & $4 / 361(1.1)^{\mathrm{a}}$ & $10 / 360(2.8)^{\mathrm{a}}$ & $3 / 420(0.7)^{\mathrm{a}}$ \\
\hline \multicolumn{5}{|c|}{$\begin{array}{l}\text { *Explanation of piglet groups: CC, both mother and piglet unvacci- } \\
\text { nated; CV, mother unvaccinated/piglet vaccinated; VC, mother vac- } \\
\text { cinated/piglet unvaccinated; VV, both mother and piglet vaccinated. } \\
\text { **NS, nursery stage }(26-63 \text { days); GS, growing stage }(64-112 \text { days); } \\
\text { FS, finishing stage }(113-161 \text { days). } \\
\text { a,b Mean values within the same row, with different superscripts, differ } \\
\text { significantly }(P<0.05) \text {. }\end{array}$} \\
\hline
\end{tabular}

Table 2. Mortality across the experimental groups

\begin{tabular}{|c|c|c|c|c|}
\hline & \multicolumn{4}{|c|}{ Number of dead pigs/number of total pigs $(\%)$} \\
\hline & $\mathrm{CC}^{*}$ & $\mathrm{CV}$ & $\mathrm{VC}$ & VV \\
\hline NS** & $5 / 282(1.8)^{\mathrm{a}}$ & $4 / 380(1.1)^{\mathrm{a}}$ & $5 / 399(1.3)^{\mathrm{a}}$ & $1 / 427(0.2)^{\mathrm{a}}$ \\
\hline GS & $32 / 277(11.6)^{\mathrm{a}}$ & $15 / 376(4.0)^{\mathrm{b}}$ & $34 / 394(8.7)^{\mathrm{ab}}$ & $6 / 426(1.4)^{b}$ \\
\hline FS & $1 / 245(0.4)^{a}$ & $0 / 361(0.0)^{\mathrm{a}}$ & $0 / 360(0.00)^{\mathrm{a}}$ & $1 / 420(0.2)^{\mathrm{a}}$ \\
\hline
\end{tabular}

*Explanation of piglet groups: CC, both mother and piglet unvaccinated; CV, mother unvaccinated/piglet vaccinated; VC, mother vaccinated/piglet unvaccinated; VV, both mother and piglet vaccinated. **NS, nursery stage (26-63 days); GS, growing stage (64-112 days); FS, finishing stage (113-161 days).

a,b Mean values within the same row, with different superscripts, differ significantly $(P<0.05)$.

Scours caused by $E$. coli were detected sporadically in a small number of pigs $(<2.5 \%)$ in all groups at the beginning of the nursery stage. Laboratory examination showed about one third of these cases were attributed to enterotoxigenic E. coli F4 strains and a quarter of them to F5 strains. Some unclassified diarrhoea was also detected in both nursery $(<1.1 \%)$ and growing stages $(<3.6 \%)$.

Mortality was markedly increased during the growing stage compared with the other stages. In that period, the CV group of PRRS-vaccinated pigs exhibited mortality three times lower, and the PRRS-vaccinated VV group, mortality eight times lower than with the CC group (Table 2). Following postmortem examination, two deaths during the nursery stage (in $\mathrm{CV}$ - and VC-groups) were attributed to severe E. coli infection, and one death during the finishing stage (in VV group) was attributed to complicated $M$. hyopneumoniae infection.

The VV pigs gained significantly more weight daily throughout all the fattening sub-periods compared with the pigs of the CC group, the latter showing the least gain $(P<0.05)$ (Table 3). When ADG was calculated for the entire fattening period, the pigs of the VV group were the best performers, followed by those of the $\mathrm{CV}$ group $(P<0.05)$ and to a lesser degree those of the VC group $(P>0.05)$. The vaccinated piglets in $\mathrm{CV}$ - and VV-groups were significantly heavier (3.3 and $4.4 \mathrm{~kg}$ respectively) at the end of the finishing stage than those of the CC group $(P<0.05)$.

The pigs of all groups had similar ADFI during all the subperiods $(P>0.05)$. However, the FCR in the VV group was the most significantly improved during each stage as well as over the entire fattening period, when compared with the CC group, followed by the $\mathrm{CV}$ group $(P<0.05)$. The $\mathrm{VC}$ group also showed improvement of FCR during some stages, but this was less than those of VV- and CV-groups (Table 3).

\section{Discussion}

Several researchers have studied dual infections of PRRSV with other pathogens (swine influenza virus, Hemophilus parasuis, Actinobacillus pleuropneumoniae, Pasteurella multocida) and have concluded that the clinical expression of combined infections is more pronounced than that observed by either pathogen alone (Carvalho et al., 1997; Pol et al., 1997; Solano et al., 1997; Van Reeth et al., 2001). This seems reasonable as PRRSV affects alveolar macrophages and interferes with local immunity, thus facilitating secondary 
Table 3. Growth performance in the groups of pigs at different stages

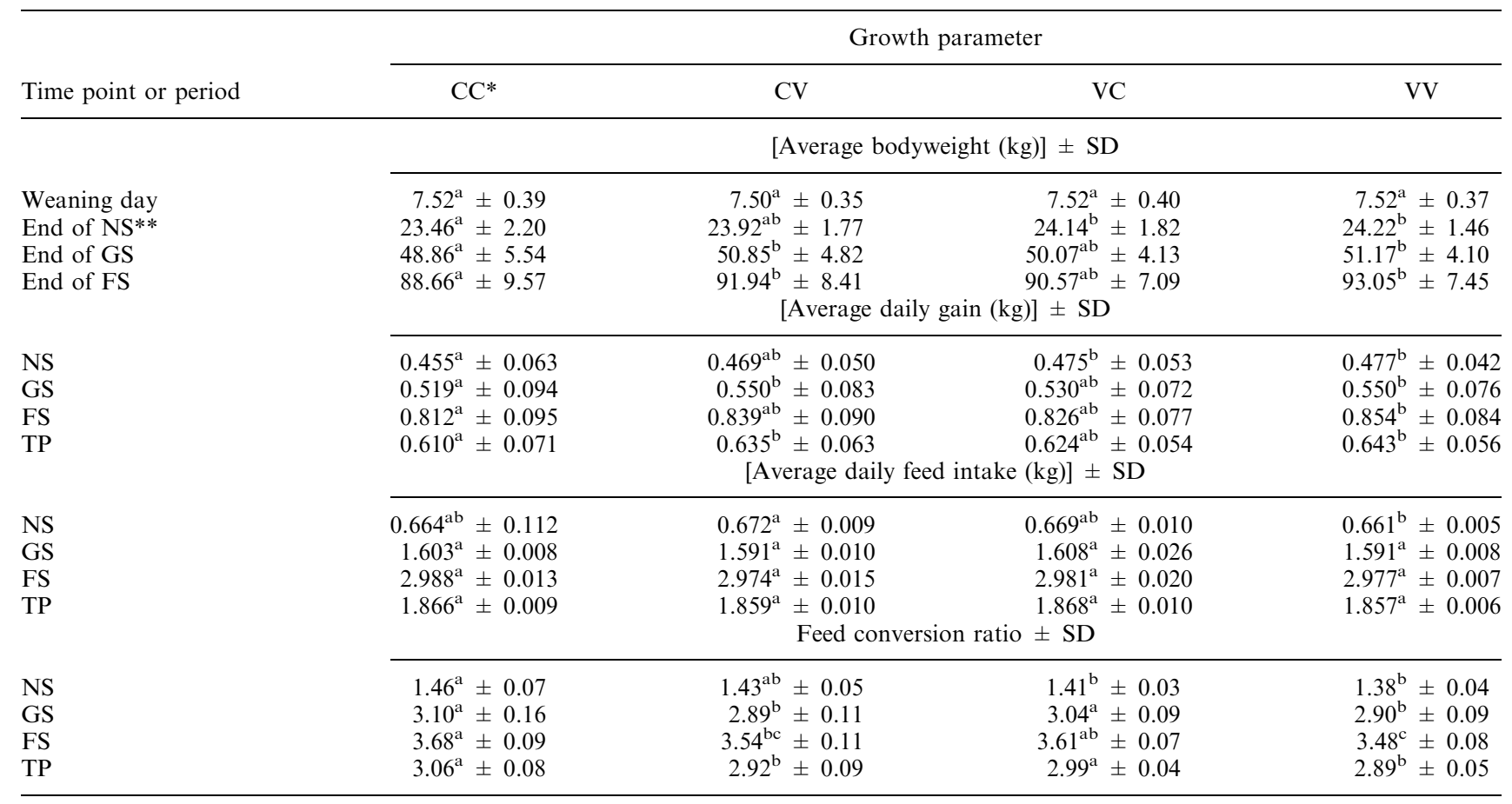

*Explanation of piglet groups: CC, both mother and piglet unvaccinated; CV, mother unvaccinated/piglet vaccinated; VC, mother vaccinated/ piglet unvaccinated; VV, both mother and piglet vaccinated.

**NS, nursery stage (26-63 days); GS, growing stage (64-112 days); FS, finishing stage (113-161 days).

Mean values within the same row, with different superscripts, differ significantly $(P<0.05)$.

infections (Benfield et al., 1999). As PRRS and PCV2 viruses are widespread in several areas worldwide, it is not uncommon to find both infections in the same populations at the same time (Rose et al., 2003; Ellis et al., 2004; Wellenberg et al., 2004). In a previous trial, performed on the same farm, the efficacy of Porcilis PRRS ${ }^{\circledR}$, was investigated with respect to sow performance (Alexopoulos et al., 2005). In that study, the beneficial effect of PRRS vaccination was demonstrated by fewer pre-mature farrowings and returns-to-oestrus, less culling, a lower incidence of postpartum dysgalactia syndrome, and by better farrowing rates, and greater numbers of pigs born alive and weaned per litter. Many of these effects explain the large difference in the starting numbers of weaned pigs between the groups of unvaccinated and vaccinated sows at the start of this study (662 and 826 weaned pigs respectively). Differences between CC- and CV-groups, or VC- and VV-groups were mainly due to meeting the parity requirement during allocation to the experimental groups. Although no sequencing data showing homology between the farm and vaccine strain were available, evidence for the dominance of an European strain in the farm and equally to both groups was, at least, convincing by indirect immunofluorescent examination.

The beneficial effect of Porcilis PRRS vaccine against the respiratory manifestations of the syndrome has already been documented in the fattening pigs of a PRRS-infected farm (Mavromatis et al., 1999). Vaccinated pigs tended to have a more rapid antibody response, and they showed reduced morbidity and mortality, with a lower incidence of respiratory bacterial infection compared with untreated pigs. In the present study, reduced morbidity and mortality was also observed in the vaccinated pigs (CV- and VV-groups) compared with the unvaccinated ones (CC- and VC-groups), suggesting that in dual infected farms, PRRSV still plays a major role in the clinical manifestation during the fattening stages, and needs to be controlled on such farms. Several clinical signs observed mainly at the end of the nursery stage and during the growing stage were compatible with those seen in PMWS. PRRS vaccination had also reduced some of these signs, a finding showing the close interaction that these two pathogens might have in the field. Reducing both PRRS and PMWS on the farm means reduced treatment costs and lower capital losses for the enterprise. Unfortunately as vaccine strain is not marked, it was not possible to exclude spread of the attenuated strain between groups of pigs, but only to avoid mechanical spread or spread by not mixing pigs, or mostly by placing groups in different airspace.

Growth parameters are not only useful for monitoring economic performance but also for the evaluation of animal health, particularly in diseases of a chronic or mild character in which visible clinical signs are absent. In this study, bodyweight, ADG and FCR of treated animals were greatly improved compared with the untreated $\mathrm{CC}$ group. It was also possible to rank the groups, with the VV group being the best performer (improvements of 5.0\% in BW, 5.4\% in ADG and $5.6 \%$ in FCR), followed by the CV group (improvements of $3.7 \%$ in $\mathrm{BW}, 4.1 \%$ in $\mathrm{ADG}$ and $4.6 \%$ in FCR $)(P<0.05)$ and to a lesser degree the $\mathrm{VC}$ group (improvements of $2.2 \%$ in $\mathrm{BW}$, $2.3 \%$ in ADG and $2.3 \%$ in FCR $)(P>0.05)$, when compared with the CC group. Since vaccination of sows conferred a beneficial effect on their offspring (Alexopoulos et al., 2005), and as vaccination of young pigs (e.g. CV group) improves 
their health and productivity, it seems reasonable that, in PRRSV- and PCV-2-infected farms, vaccination of both sows and their offspring produces an almost additive effect in delivering the best health and productivity results.

\section{References}

Alexopoulos, C., S. K. Kritas, C. S. Kyriakis, E. Tzika, and S. C. Kyriakis, 2005: Sow performance in an endemically porcine reproductive and respiratory syndrome (PRRS)-infected farm after sow vaccination with an attenuated PRRS vaccine. Vet. Microbiol. 111, 151-157.

Benfield, D. A., J. E. Collins, S. A. Dee, P. G. Halbur, H. S. Joo, K. M. Lager, W. L. Mengeling, M. P. Murtaugh, K. D. Rossow, G. W. Stevenson, and J. J. Zimmerman, 1999: Porcine reproductive and respiratory syndrome. In: Straw, B. E., S. D'Allaire, W. L. Mengeling, and D. J. Taylor (eds), Diseases of Swine, 8th edn, pp. 201-232. Iowa State University Press, Ames, IA, USA.

Carvalho, L. F., J. Segales, and C. Pijoan, 1997: Effect of porcine reproductive and respiratory syndrome virus on subsequent Pasteurella multocida challenge in pigs. Vet. Microbiol. 55, 241-246.

Dea, S., C. A. Gagnon, H. Mardassi, and G. Milane, 1996: Antigenic variability among North American and European strains of porcine reproductive and respiratory syndrome virus as defined by monoclonal antibodies to the matrix protein. J. Clin. Microbiol. 34, 1488-1493.

Dee, S. A., 2003: Principles of prevention, control and eradication. In: Zimmerman, J., K.-J. Yoon, and E. Neumann (eds), PRRS Compendium: A Reference for Pork Producers, pp. 79-87. National Pork Board, Des Moines, IA, USA

Dee, S. A., and R. E. Philips, 1999: Use of polymerase chain reaction to detect vertical transmission of PRRS virus in piglets from gilt litters. Swine Health Prod. 7, 237-239.

Ellis, J., E. Clark, D. Haines, K. West, S. Krakowka, S. Kennedy, and G. M. Allan, 2004: Porcine circovirus-2 and concurrent infections in the field. Vet. Microbiol. 98, 159-163.

Keffaber, K. K., 1989: Reproductive failure of unknown etiology. Am. Assoc. Swine Pract. News 1, 1-10.

Kritas, S. K., A. R. Burriel, A. H. Tzivara, A. Govaris, S. C. Kyriakis, H. Karatzias, and J. Vlemmas, 2003: Prevention of scours in neonatal kids after modification of management and experimental vaccination against Escherichia coli. Small Rumin. Res. 50, 51-56.

Labarque, G., K. Van Reeth, H. Nauwynck, C. Drexler, S. Van Gucht, and M. Pensaert, 2004: Impact of genetic diversity of Europeantype porcine reproductive and respiratory syndrome virus strains on vaccine efficacy. Vaccine 22, 4183-4190.

Lukert, P. D., and G. M. Allan, 1999: Porcine Circovirus. In: Straw, B. E., S. D'Allaire, W. L. Mengeling, and D. J. Taylor (eds), Diseases of Swine, $8^{\text {th }}$ edn, pp. 119-124. Iowa State University Press, Ames, IA, USA.

Magar, R., R. Larochelle, S. Dea, C. A. Gagnon, E. A. Nelson, J. Christopher-Hennings, and D. A. Benfield, 1995: Antigenic comparison of Canadian and US isolates of porcine reproductive and respiratory syndrome virus using monoclonal antibodies to the nucleocapsid protein. Can. J. Vet. Res. 59, 232-234.

Mavromatis, I., S. K. Kritas, C. Alexopoulos, A. Tsinas, and S. C. Kyriakis, 1999: Field evaluation of a live vaccine against porcine reproductive and respiratory syndrome in fattening pigs. J. Vet. Med. B 46, 603-612.

Meredith, M. J., 1995: Porcine Reproductive and Respiratory Syndrome (PRRS), 1st European edn, pp. 5-10. Pig Disease Information Centre, Department of Clinical Veterinary Medicine, University of Cambridge, Boehringer, Ingelheim.

Murakami, Y., A. Kato, T. Tsuda, T. Morozumi, Y. Miura, and T. Sugimura, 1994: Isolation and serological characterization of porcine reproductive and respiratory syndrome (PRRS) viruses from pigs with reproductive and respiratory disorders in Japan. J. Vet. Med. Sci. 56, 891-894.

Pol, J. M., L. A. van Leengoed, N. Stockhofe, G. Kok, and G. Wensvoort, 1997: Dual infections of PRRSV/influenza or PRRSV/ Actinobacillus pleuropneumoniae in the respiratory tract. Vet. Microbiol. 55, 259-264.

Polson, D. D., W. E. Marsh, and G. D. Dial, 1992: Financial evaluation and decision making in the swine breeding herd. Vet. Clin. North Am. Food Anim. Pract. 8, 725-747.

Rose, N., G. Larour, G. Le Diguerher, E. Eveno, J. P. Jolly, P. Blanchard, A. Oger, M. Le Dimna, A. Jestin, and F. Madec, 2003: Risk factors for porcine post-weaning multisystemic wasting syndrome (PMWS) in 149 French farrow-to-finish herds. Prev. Vet. Med. 61, 209-225.

Rovira, A., M. Balasch, J. Segales, L. Garcia, J. Plana-Duran, C. Rosell, H. Ellerbrok, A. Mankertz, and M. Domingo, 2002: Experimental inoculation of conventional pigs with porcine reproductive and respiratory syndrome virus and porcine circovirus 2 . J. Virol. 76, 3232-3239.

Solano, G. I., J. Segales, J. E. Collins, T. W. Molitor, and C. Pijoan, 1997: Porcine reproductive and respiratory syndrome virus (PRRSv) interaction with Haemophilus parasuis. Vet. Microbiol. 55, 247-257.

Van Reeth, K., H. Nauwynck, and M. Pensaert, 2001: Clinical effects of experimental dual infections with porcine reproductive and respiratory syndrome virus followed by swine influenza virus in conventional and colostrums-deprived pigs. J. Vet. Med. B Infect. Dis. Vet. Public Health 48, 283-292.

Wellenberg, G. J., N. Stockhofe-Zurwieden, W. J. Boersma, M. F. De Jong, and A. R. Elbers, 2004: The presence of co-infections in pigs with clinical signs of PMWS in the Netherlands: a case-control study. Res. Vet. Sci. 77, 177-184.

Wensvoort, G., C. Terpstra, J. M. Pol, E. A. ter Laak, M. Bloemraad, E. P. De Kluyver, C. Kragten, L. van Buiten, A. Den Besten, F. Wagenaar, J. M. Broekhuijsen, P. L. J. M. Moonen, T. Zetstra, E. A. De Boer, H. J. Tibben, M. F. De Jong, P. Van' t Veld, G. J. R. Groenland, J. A. van Gennep, M. T. H. Voets, J. H. M. Verheijden, and J. Braamskamp, 1991: Mystery swine disease in the Netherlands: the isolation of Lelystad virus. Vet. Q. 13, 121-130.

Wensvoort, G., E. P. De Kluyver, E. A. Luijtze, den Besten, A., L. Harris, J. E. Collins, W. T. Christianson, and D. Chladek, 1992: Antigenic comparison of Lelystad virus and swine infertility and respiratory syndrome (SIRS) virus. J. Vet. Diagn. Invest. 4, 134-138.

Yoon, K.-J., J. J. Zimmerman, S. L. Swenson, M. J. McGinley, K. A. Eernisse, A. Brevik, L. L. Rhinehart, M. L. Frey, H. T. Hill, and K. B. Platt, 1995: Characterization of the humoral immune response to porcine reproductive and respiratory syndrome (PRRS) virus infection. J. Vet. Diagn. Invest. 7, 305-312. 